\title{
Direct Electrochemistry of Horseradish Peroxidase on NiO Nanoflower Modified Electrode and Its Electrocatalytic Activity
}

\author{
Lijun Yan, ${ }^{1}$ Xiaofeng Wang, ${ }^{2}$ Qiutong Li, ${ }^{1}$ Wencheng Wang, ${ }^{1}$ Shixing Gong, ${ }^{1}$ Zheng You, ${ }^{2}$ Peng Yi, ${ }^{1}$ Wei Sun ${ }^{1, *}$
}

\author{
1 Key Laboratory of Tropical Medicinal Plant Chemistry of Ministry of Education, College of Chemistry and Chemical Engineering, Hainan Normal University, \\ Haikou 571158, P. R. China \\ 2 Department of Precision Instruments, Tsinghua University, Beijing 100084, P. R. China \\ * Corresponding author's e-mail address: swyy26@hotmail.com
}

RECEIVED: August 15, 2015 * REVISED: October 2, 2016 * ACCEPTED: October 3, 2016

\begin{abstract}
In this paper nickel oxide $(\mathrm{NiO})$ nanoflower was synthesized and used for the realization of direct electrochemistry of horseradish peroxidase (HRP). By using carbon ionic liquid electrode (CILE) as the substrate electrode, NiO-HRP composite was casted on the surface of CILE with chitosan (CTS) as the film forming material and the modified electrode was denoted as CTS/NiO-HRP/CILE. UV-Vis absorption and FT-IR spectra confirmed that HRP retained its native structure after mixed with NiO nanoflower. Direct electron transfer of HRP on the modified electrode was investigated by cyclic voltammetry with a pair of quasi-reversible redox waves appeared, indicating that the presence of $\mathrm{NiO}$ nanoflower on the electrode surface could accelerate the electron transfer rate between the electroactive center of HRP and the substrate electrode. Electrochemical behaviors of HRP on the modified electrode were carefully investigated. The HRP modified electrode showed excellent electrocatalytic activity to the reduction of trichloroacetic acid with wider linear range and lower detection limit. Therefore the presence of $\mathrm{NiO}$ nanoflower could provide a friendly biocompatible interface for immobilizing biomolecules and keeping their native structure. The fabricated electrochemical biosensor displayed the advantages such as high sensitivity, good reproducibility and long-term stability.
\end{abstract}

Keywords: nickel oxide nanoflower, horseradish peroxidase, carbon ionic liquid electrode, direct electrochemistry, electrocatalysis.

\section{INTRODUCTION}

D IRECT electron transfer between redox proteins and enzymes with the electrode is of great significance for the understanding the electron transfer process in biological system. ${ }^{[1]}$ The results can also be used for the development of third-generation biosensor and bioreactor without the usage of mediators. ${ }^{[2]}$ However the electroactive centers of redox proteins are often buried deeply in the molecular structure of redox proteins, and the distances between the redox centers and electrode are increased. Also the unfavorable orientation of redox proteins can result in a slow electron transfer efficiency. ${ }^{[3]}$ Greart efforts have been proposed to facilitate the electron transfer of redox proteins with the usage of nanomaterials. Nanosized materials exhibit the advantages including biocompatibility, large surface area and good conductivity, which can provide a suitable microenvironment for redox proteins to exchange electrons. ${ }^{[4]}$

Among nanomaterials used nanosized metal oxide can act as suitable matrix for the immobilization of redox proteins, which exhibit the characteristics including wide electrochemical working window, good biocompatibility, large surface area, high electrical conductivity, chemical and photochemical stability, ease of preparation and excellent substrate adhesion. As a semiconductive metal oxide with a high isoelectric point of $10.5,{ }^{[5]} \mathrm{NiO}$ nanoparticle has been used in the preparation of electrochemical sensors. It has been reported that $\mathrm{NiO}$ nanomaterials have exhibited excellent catalytic, magnetic, optical and electrochemical characteristics. ${ }^{[6,7]}$ Also $\mathrm{NiO}$ nanomaterials have the properties such as good biocompatibility, electroinactivity in physiological $\mathrm{pH}$ solution and high porosity, which can be used for the immobilization of different biomolecules with 
the electron transfer accelerated. Noorbakhsh et al. fabricated an electrochemical DNA biosensor by immobilizing ssDNA on NiO nanoparticle modified glassy carbon electrode (GCE). ${ }^{[8]}$ Mohan et al. applied a nanostructure NiO modified indium tin oxide electrode for the electrochemical detection of specific sequence of visceral leishmaniasis. ${ }^{[9]}$ Salimi et al. immobilized copper and zinc-superoxide dismutase onto electrodeposited $\mathrm{NiO}$ nanoparticle modified GCE to fabricate an amperometric superoxide biosensor. ${ }^{[10]}$ $\mathrm{Mu}$ et al. applied a nanosized NiO modified carbon paste electrode for non-enzymatic glucose sensor. ${ }^{[11]}$

As a new kind of working electrode, carbon ionic liquid electrode (CILE) has been used in the preparation of electrochemical sensor. ${ }^{[12]}$ CILE is prepared by incorporating ionic liquid (IL) as the binder and the modifier in traditional carbon paste electrode (CPE), which has been elucidated with the advantages such as easy preparation, good reversibility, high sensitivity and the ability to lower the overpotential of electroactive compounds. ${ }^{[13]}$ CILE can also be decorated to get the modified electrode. Different kinds of the modified CILE had been prepared and used for electrochemical sensing with excellent performance, which served as the basal electrode for the further modification. ${ }^{[14-16]}$

In this paper $\mathrm{NiO}$ nanoflower was synthesized by a hydrothermal method and further used for the electrode modification. Horseradish peroxidase (HRP) was immobilized with $\mathrm{NiO}$ nanoflower and direct electrochemistry of HRP was realized on the modified electrode. The NiO-HRP composite modified electrode showed excellent electrocatalytic activity to the reduction of trichloroacetic acid (TCA).

\section{EXPERIMENTAL}

\section{Reagents}

HRP (Shanghai Xueman Biotech. Ltd. Co., China), graphite powder (Shanghai Colloid Chem. Co., particle size of $30 \mu \mathrm{m}$ ) 1-hexylpyridinium hexafluorophosphate $\left(\mathrm{HPPF}_{6}\right.$, > $99 \%$, Lanzhou Greenchem. ILS. LICP. CAS., China), chitosan (CTS, Dalian Xindie Ltd. Co., China) and TCA (Tianjin Kemiou Chem. Ltd. Co., China) were used as received. $0.1 \mathrm{~mol} \mathrm{~L}^{-1}$ phosphate buffer solutions (PBS) with various $\mathrm{pH}$ values were used as the supporting electrolyte. All the other chemicals used were of analytical reagent grade and doubly distilled water was used in the experiments.

\section{Apparatus}

Voltammetric measurements were executed on a $\mathrm{CHI}$ 1210A electrochemical workstation (Shanghai CH Instrument, China). Electrochemical impedance spectroscopy (EIS) was performed on a CHI 660D electrochemical workstation (Shanghai $\mathrm{CH}$ Instrument, China) in a $0.1 \mathrm{~mol} \mathrm{~L}^{-1} \mathrm{KCl}$ solution containing $1.0 \mathrm{mmol} \mathrm{L}^{-1}\left[\mathrm{Fe}(\mathrm{CN})_{6}\right]^{3-/ 4-}$ with the frequencies swept from $10^{5}$ to $10^{-1} \mathrm{~Hz}$. The AC voltage amplitude was set as $5 \mathrm{mV}$ and the applied potential was $202 \mathrm{mV}$. A conventional three-electrode system was used with a HRP modified electrode as the working electrode, a platinum wire as the auxiliary electrode and a saturated calomel electrode (SCE) as the reference electrode. FT-IR spectra and UV-Vis absorption spectra were recorded on Nicolet 6700 FT-IR spectrophotometer (Thermo Fisher Scientific Inc., USA) and TU-1901 double beam UV-Visible spectrophotometer (Beijing General Instrument Ltd. Co., China). Scanning electron microscopy (SEM) was recorded on a JSM-7100F scanning electron microscope (Japan Electron Company, Japan).

\section{Synthesis of NiO Nanoflower}

$\mathrm{NiO}$ nanoflower was synthesized based on the reported procedure. ${ }^{[17]} \mathrm{In}$ brief $0.2 \mathrm{~g}$ of sulfonated polystyrene was dispersed into a solution containing $20 \mathrm{~mL}$ of deionized water and $20 \mathrm{~mL}$ of ethanol by sonication for 10 minutes. Then $0.4 \mathrm{~g}$ of nickel nitrate hexahydrate $\left(\mathrm{Ni}\left(\mathrm{NO}_{3}\right)_{2} \cdot 6 \mathrm{H}_{2} \mathrm{O}\right)$ and 1.2 $\mathrm{g}$ of urea were added under stirring for 10 minutes. The flask was transferred into an $80^{\circ} \mathrm{C}$ oil bath and kept for 12 h. After that the product was collected via centrifugation at $8000 \mathrm{rpm}$ for 6 minutes, which was further washed with water and ethanol for three cycles of centrifugation/redispersion. The products were dried in an $80^{\circ} \mathrm{C}$ oven for $12 \mathrm{~h}$ to get sulfonated polystyrene@ $\mathrm{Ni}_{2}-\mathrm{CO}_{3}(\mathrm{OH})_{2}$, which was converted into $\mathrm{NiO}$ nanoflower by calcining at $450{ }^{\circ} \mathrm{C}$ for $2 \mathrm{~h}$ in air at a ramping rate of $2^{\circ} \mathrm{C} \mathrm{min}^{-1}$.

\section{Preparation of the Modified Electrode}

CILE was fabricated according to a reported procedure. ${ }^{[18]}$ In brief the mixture of $1.6 \mathrm{~g}$ graphite powder and $0.8 \mathrm{~g}$ $\mathrm{HPPF}_{6}$ was packed into a cavity of a glass tube $(\Phi=4.0 \mathrm{~mm})$. Then a copper wire was inserted to establish an electrical contact. Prior to use the electrode was polished on a weighing paper to get a mirror-like surface.

A mixture solution contained $10.0 \mathrm{mg} \mathrm{mL}^{-1} \mathrm{HRP}$ and $1.0 \mathrm{mg} \mathrm{mL}^{-1} \mathrm{NiO}$ nanoflower was prepared. Then $8.0 \mu \mathrm{L}$ of the mixture was casted on the surface of CILE. After dried at room temperature, $5.0 \mu \mathrm{L}$ of $1.0 \mathrm{mg} \mathrm{mL}^{-1} \mathrm{CTS}$ solution (in $1.0 \%$ HAc solution) was dropped on the electrode to form a stable film. The resulted electrode was denoted as $\mathrm{CTS} / \mathrm{NiO}-\mathrm{HRP} / \mathrm{CILE}$ and stored at $4{ }^{\circ} \mathrm{C}$ when not in use. Other modified electrodes such as CTS/HRP/CILE, $\mathrm{CTS} / \mathrm{NiO} / \mathrm{CILE}$ etc. were prepared by the similar procedures and used for the comparison.

\section{Procedure}

Electrochemical measurements were performed in a $10 \mathrm{~mL}$ electrochemical cell containing $0.1 \mathrm{~mol} \mathrm{~L}^{-1} \mathrm{PBS}$, which was purged with highly purified nitrogen for $30 \mathrm{~min}$ prior to experiments with a nitrogen atmosphere maintained 
during the experiments. UV-Vis spectroscopic experiments were performed with a mixture solution of HRP and $\mathrm{NiO}$ nanoflower in $\mathrm{pH}=7.0 \mathrm{PBS}$. The NiO-HRP and HRP solution were dropped on a glass slide and dried to get the film for FT-IR measurements.

\section{RESULTS AND DISCUSSION}

\section{SEM Images}

SEM image of the synthesized NiO nanomaterial was recorded with the result shown in Figure $1 \mathrm{~A}$, which exhibited a 3D flower-like microstructure with the average size of 2$3 \mu \mathrm{m}$. High-magnified SEM micrograph (Figure 1B) showed that this microstructure was assembled by plate-like nanostructure with the thickness of few nanometers. This 3D flower-like $\mathrm{NiO}$ microstructure assembled by hexagonal nanoplates can provide a porous structure for the further immobilization of HRP.

\section{Spectroscopic Results}

FT-IR spectroscopy is sensitive to the conformational changes of protein and commonly used to check the conformational integrity of the heme proteins. ${ }^{[19]}$ The shapes of amide I $\left(1700-1600 \mathrm{~cm}^{-1}\right.$, caused by $\mathrm{C}=0$ stretching vibrations of peptide linkages in the protein's backbone) and amide II (1600-1500 $\mathrm{cm}^{-1}$, attributed to the combination of $\mathrm{N}-\mathrm{H}$ bending and $\mathrm{C}-\mathrm{N}$ stretching) infrared absorption band of HRP can provide detailed information about the secondary structure of the polypeptide chain. [20] The amide I and II bands of the native state of HRP appeared at $1650 \mathrm{~cm}^{-1}$ and $1540 \mathrm{~cm}^{-1}$ (Figure 2(A)-1), which was close to that of the HRP-NiO mixture at 1651 $\mathrm{cm}^{-1}$ and $1540 \mathrm{~cm}^{-1}$ (Figure 2(A)-2). In UV-Vis absorption spectroscopy, the location of the Soret absorption band from the four iron heme groups of proteins can provide structural information about possible denaturation or the conformational change in the heme group region. ${ }^{[21]}$ As shown in Figure 2B, the characteristic Soret absorption of HRP appeared at $398.0 \mathrm{~nm}$ (curve 1), and the mixture solution of NiO-HRP gave the Soret band at the same value without changes (curve 2). All the spectroscopic results suggested that HRP retained the essential features of its native structure without any changes after mixed with NiO nanoflower. Therefore the good biocompatibility of $\mathrm{NiO}$ nanoflower could provide a biocompatible microenvironment for HRP immobilization.

\section{EIS of the Modified Electrodes}

EIS can be used to get the interfacial information of the modified electrodes. In the Nyquist diagram the semicircular portion at high frequencies corresponds to the electrontransfer-limited process and the linear part at lower
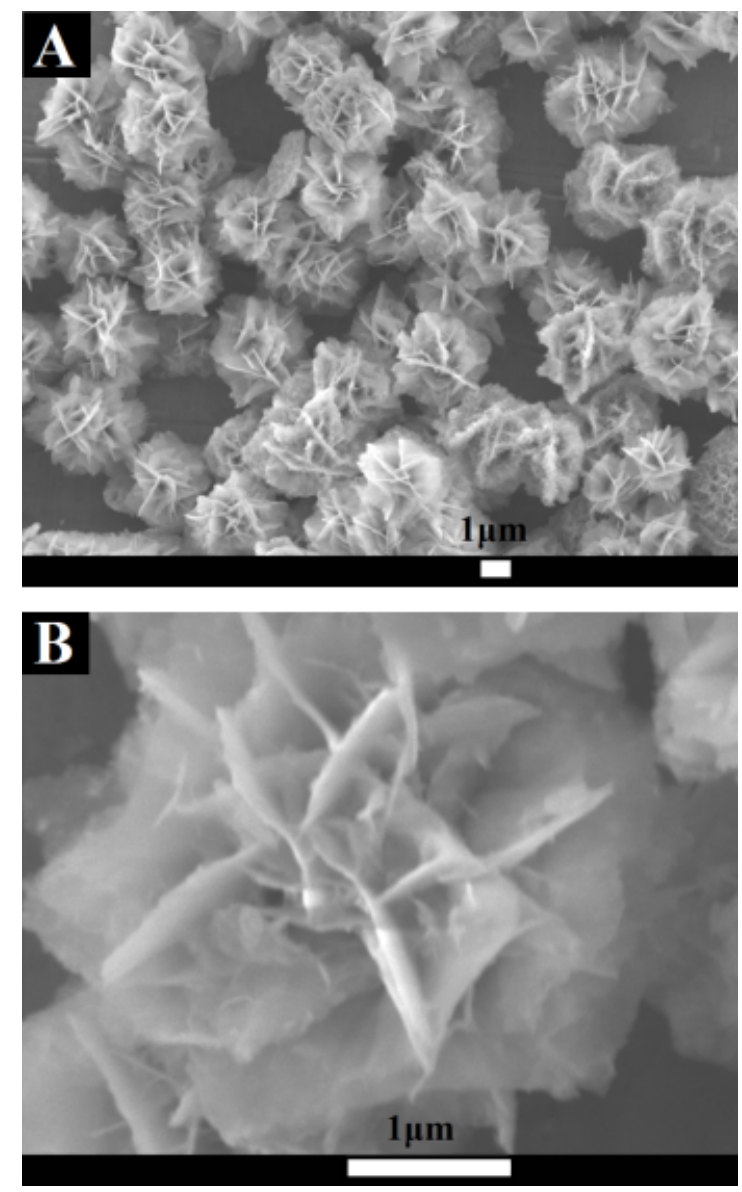

Figure 1. SEM image of $\mathrm{NiO}$ nanoflower (A) and (B) as the enlarged image.

frequencies corresponds to the diffusion process. The electron-transfer resistance (Ret) can be obtained from the diameter of semi-circular portion, which controls the electron-transfer kinetics of the redox probe at the electrode. As shown in Figure 3, the Ret value of CILE was got as 66.05 $\Omega$ (curve a) and that of CTS/NiO/CILE decreased to $43.66 \Omega$ (curve b). So the presence of $\mathrm{NiO}$ nanoflower on the electrode surface can increase the effective surface area with certain conductivity, which acts as a good electron transfer media to lower the interfacial resistance. On CTS/HRP/CILE a substantial increase in the diameter of the semicircle was observed with the Ret value as $90.09 \Omega$ (curve d). The result indicated that the presence of HRP molecules on the electrode surface could hinder the diffusion of ferricyanide toward the electrode surface, which acted as the inert electron and mass transfer blocking layer. While on CTS/NiO-HRP/CILE the diameter of Nyquist circle decreased with the Ret value as $56.57 \Omega$ (curve c). The presence of $\mathrm{NiO}$ nanoflower with HRP on the electrode can provide a large surface area and good interconnection network, which act as a good electron-transfer media between the probe and 

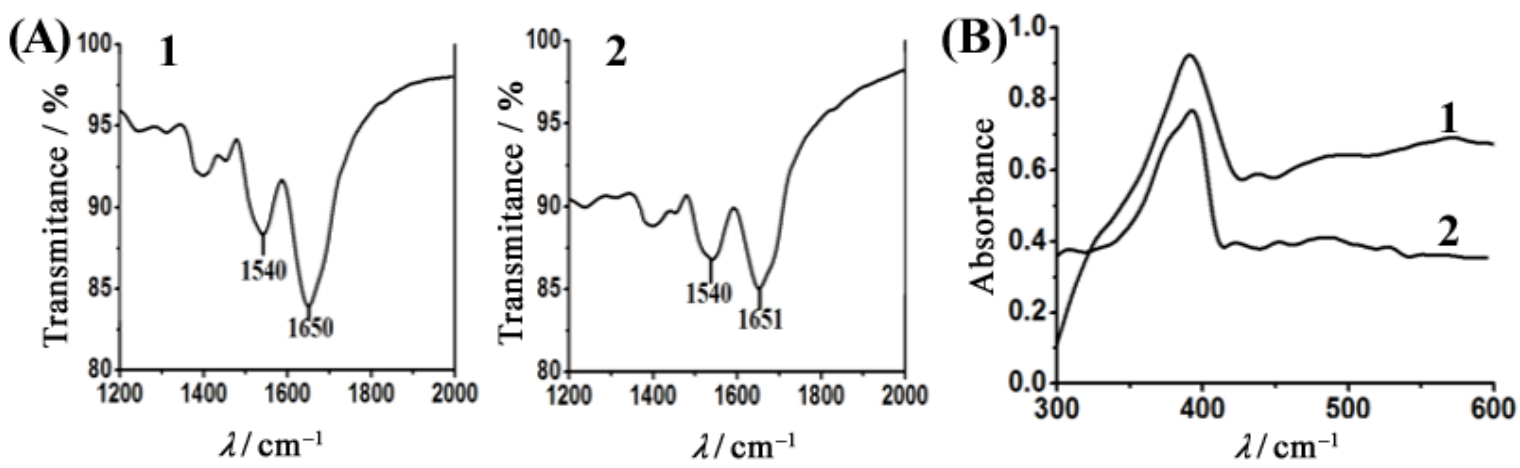

Figure 2. (A) FT-IR spectra of (1) HRP and (2) NiO-HRP; (B) UV-Vis absorption spectra of (1) HRP and (2) NiO-HRP in pH = $3.0 \mathrm{PBS}$.

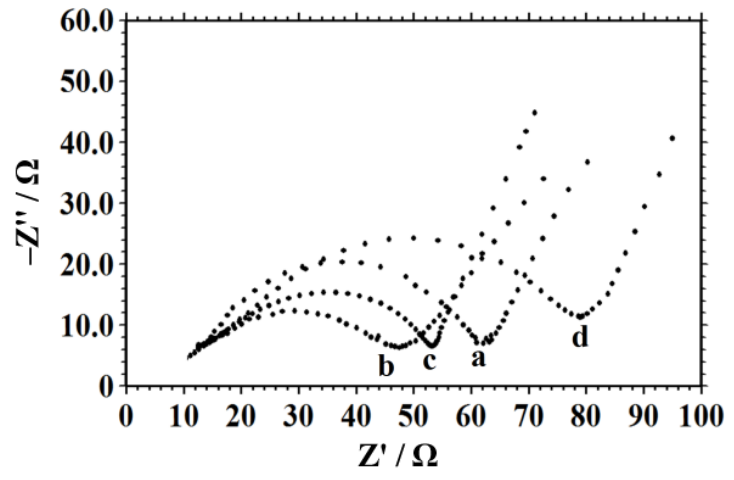

Figure 3. EIS of (a) CILE, (b) CTS/NiO/CILE, (c) CTS/NiO-HRP/CILE and (d) CTS/HRP/CILE in the presence of $1.0 \mathrm{mmol} \mathrm{L^{-1 }}$ $\left[\mathrm{Fe}(\mathrm{CN})_{6}\right]^{3-/ 4-}$ and $0.1 \mathrm{~mol} \mathrm{~L}^{-1} \mathrm{KCl}$ mixture solution.

the electrode. The gradually changes of the interfacial resistance on different electrodes indicated the successfully preparation of the modified electrodes.

\section{Direct Electrochemical Behaviors of the HRP Modified Electrode}

Electrochemical behaviors of different modified electrodes were carefully investigated in $\mathrm{pH}=3.0$ PBS with cyclic voltammogram shown in Figure 4. No electrochemical responses could be observed at CTS/CILE (curve a) or CTS/NiO/CILE (curve b), indicating no electrochemical reaction took place. On CTS/HRP/CILE a pair of unsymmetric redox peaks appeared (curve c), indicating that direct electron transfer between HRP and CILE was realized. CILE has exhibited high conductivity with biocompatible interface, which is suitable for protein to transfer electron. ${ }^{[22]}$ On CTS/NiO-HRP/CILE the redox peak currents increased greatly with the peak shape became more symmetry (curve d). Also multi-scan cyclic voltammetric results indicated that the redox peaks almost unchanged after continuous potential cycling. Therefore the presence of $\mathrm{NiO}$ nanoflower on electrode accelerated the direct electron transfer rate of HRP with good stability. NiO nanoflower is a semiconductor with large

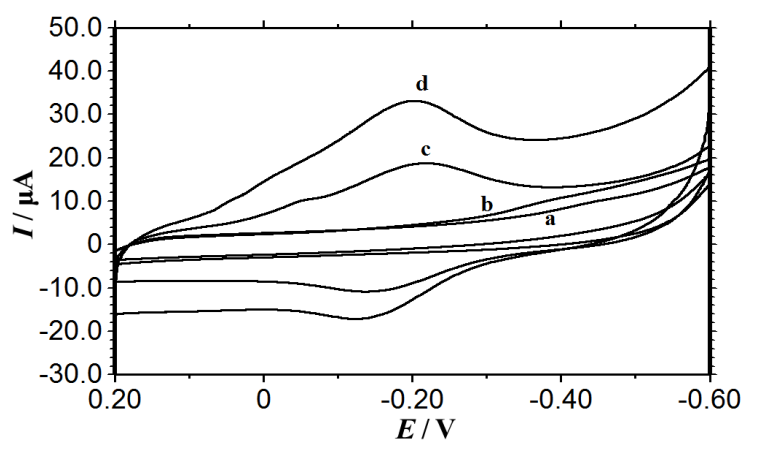

Figure 4. Cyclic voltammograms of (a) CTS/CILE, (b) $\mathrm{CTS} / \mathrm{NiO} / \mathrm{CILE}$, (c) CTS/HRP/CILE and (d) CTS/NiO-HRP/CILE in $\mathrm{pH}=3.0$ PBS with scan rate as $100 \mathrm{mV} \mathrm{s}^{-1}$.

surface area, which can act as the bridge and establish a fast electron transfer path to facilitate electron transfer of HRP with the underlying electrode. The values of cathodic peak potential $\left(E_{\mathrm{pc}}\right)$ and anodic peak potential $\left(E_{\mathrm{pa}}\right)$ in curve $\mathrm{d}$ were got as $-0.200 \mathrm{~V}$ and $-0.122 \mathrm{~V}$ with the peak-to-peak separation $\left(\Delta E_{\mathrm{p}}\right)$ as $78 \mathrm{mV}$. The formal peak potential $\left(E^{0^{\prime}}\right)$ that calculated from the midpoint of $E_{\mathrm{pa}}$ and $E_{\mathrm{pc}}$ was estimated as $-0.161 \mathrm{~V}$ (vs. SCE), which was the typical characteristic of electroactive heme $\mathrm{Fe}(\mathrm{III}) / \mathrm{Fe}(\mathrm{II})$ redox couples.[22]

\section{Electrochemical Investigations}

Electrochemical responses of this HRP modified electrode were investigated by cyclic voltammetry with the results shown in Figure 5A. A pair of well-defined quasi-reversible redox peaks could be observed on cyclic voltammograms with almost equal height of peak currents. Two linear regression equations were calculated as $I_{\mathrm{pc}} / \mu \mathrm{A}=-56.93 \mathrm{u} / \mathrm{V}$ $\mathrm{s}^{-1}-1.56(\nu=0.995)$ and $I_{\mathrm{pa}} / \mu \mathrm{A}=78.90 u / \mathrm{V} \mathrm{s}^{-1}+3.58(\gamma=$ 0.992 ) in the scan rate range from 0.05 to $0.5 \mathrm{~V} \mathrm{~s}^{-1}$ (as shown in Figure 5B), indicating a typical surface-controlled electrochemical behavior. By integration of the reduction or oxidation peak of cyclic voltammograms the surface coverage $\left(\Gamma^{*}\right)$ of electroactive HRP can be estimated from the equation $\left(\Gamma^{*}=Q / n A F\right) .{ }^{[23]}$ The result was got as $1.09 \times 10^{-9}$ 

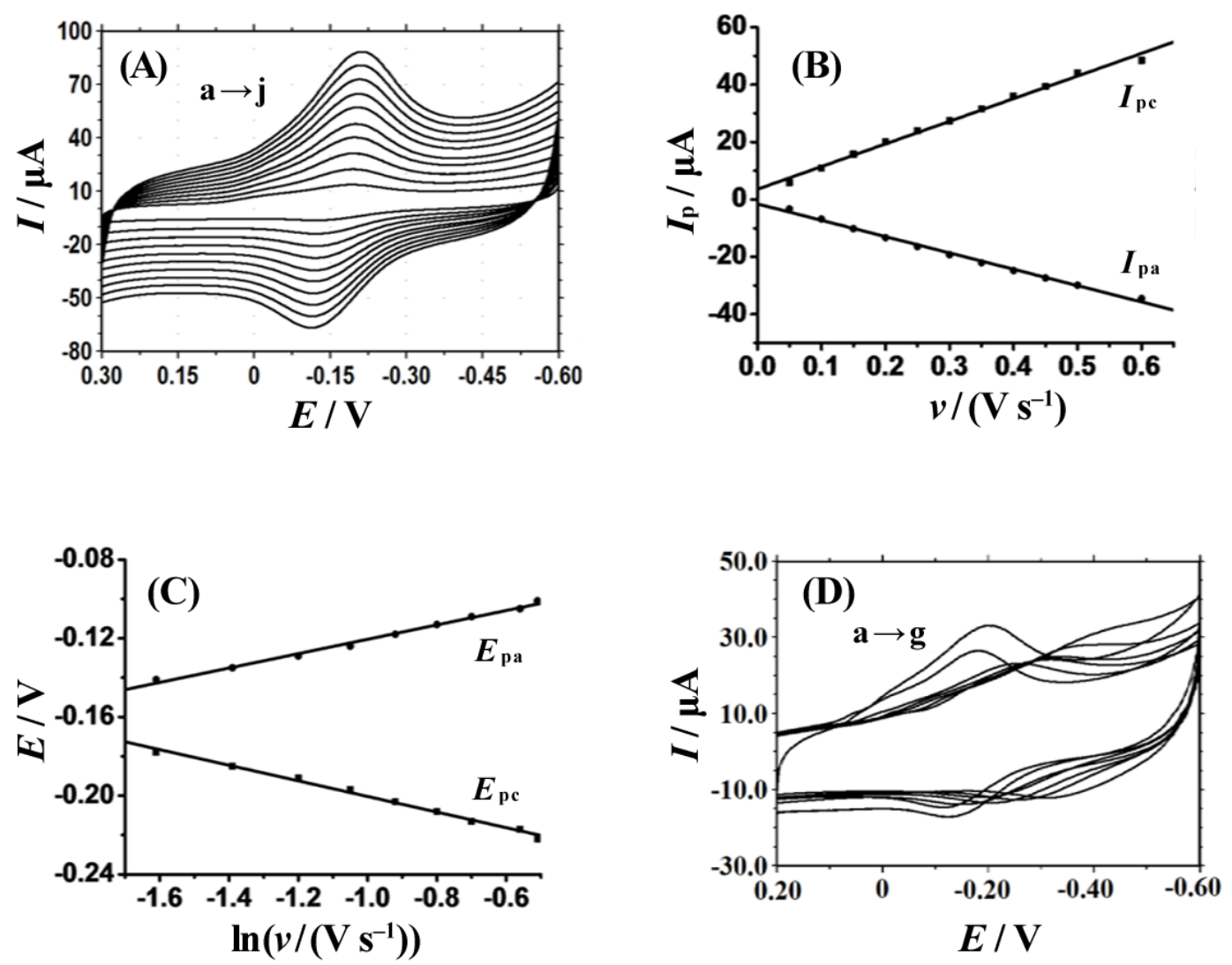

Figure 5. (A) Influence of scan rate on electrochemical responses of CTS/NiO-HRP/CILE in pH 3.0 PBS with scan rates from a to $\mathrm{j}$ as $50,100,150,200,250,300,350,400,450,500 \mathrm{mV} \mathrm{s}^{-1}$, respectively. (B) Linear relationship of redox peak currents versus scan rate (U). (C) Linear relationship of the redox peak potentials versus InU. (D) Cyclic voltammograms of CTS/NiO-HRP/CILE in different $\mathrm{pH}$ PBS (from a to $g$ as 2, 3, 4, 5, 6, 7, 8) with the scan rate of $100 \mathrm{mV} \mathrm{s}^{-1}$.

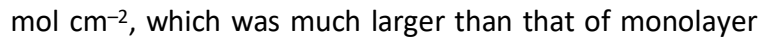
coverage $\left(1.89 \times 10^{-11} \mathrm{~mol} \mathrm{~cm}^{-2}\right)$. ${ }^{[24]}$ While the total amount of HRP on the electrode surface was calculated as $1.59 \times 10^{-8} \mathrm{~mol} \mathrm{~cm}^{-2}$, so the fraction of electroactive HRP among the total HRP on the electrode was got as $6.85 \%$. The results indicated that multilayers of HRP in the composite close to the electrode with suitable orientation could exchange electron with electrode. The increase of scan rate also resulted in the shift of the redox peak potentials gradually with the increase of the peak-to-peak separation. As shown in Figure 5C, the relationships of $\mathrm{Ep}$ with Inu were constructed with two equations as $E_{\mathrm{pc}} / \mathrm{V}=-0.040 \operatorname{lnu}-0.24$ $(\gamma=0.991)$ and $E_{\mathrm{pa}} / \mathrm{V}=0.037 \ln u-0.084(\gamma=0.992)$. According to the Laviron's equations, ${ }^{[25]}$ the values of the electron transfer coefficient $(\alpha)$, the electron transfer number $(n)$ and the heterogeneous electron transfer rate constant $\left(k_{\mathrm{s}}\right)$ were estimated as $0.64,0.97$ and $2.97 \mathrm{~s}^{-1}$, respectively. The $k_{\mathrm{s}}$ value of $2.97 \mathrm{~s}^{-1}$ was much larger than that of $0.97 \mathrm{~s}^{-1}$ at Nafion/Hb-GR-MWCNT/CILE, [26] $1.14 \mathrm{~s}^{-1}$

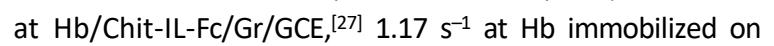

$\mathrm{SiO}_{2}$ /AuNPs/ACNTs. ${ }^{[28]}$ This $k_{\mathrm{s}}$ value indicated that the presence of $\mathrm{NiO}$ nanoflower could form a suitable microenvironment that benefit the electron transfer of HRP with electrode.

The effect of buffer $\mathrm{pH}$ on electrochemical responses of CTS/NiO-HRP/CILE was further investigated in the $\mathrm{pH}$ range from 3.0 to 8.0 with the results shown in Figure 5D. With the increase of buffer $\mathrm{pH}$ a pair of redox peaks appeared and deformed gradually along with the negatively shift of the redox peak potentials, implying that protons were involved in the electrode reaction. The formal peak potential $\left(E^{0^{\prime}}\right)$ had good linear relationship with buffer $\mathrm{pH}$ and the regression equation was $E^{\prime} / \mathrm{V}=-0.045 \mathrm{pH}-0.019$ $(\gamma=0.996)$. The slope value of $-45.0 \mathrm{mV} \mathrm{pH}^{-1}$ was a little smaller than the theoretical value of $-59.0 \mathrm{mV} \mathrm{pH}^{-1}$ at $25^{\circ} \mathrm{C}$ for a single-proton coupled one-electron transfer process. Therefore the electrochemical reaction could be expressed with the equation as: HRP heme Fe (III) $+\mathrm{H}^{+}+\mathrm{e}^{-} \leftrightarrow$ HRP heme Fe (II). Also the biggest redox peak currents appeared at $\mathrm{pH}=3.0$ buffer solution, which was selected for the electrochemical investigation. 

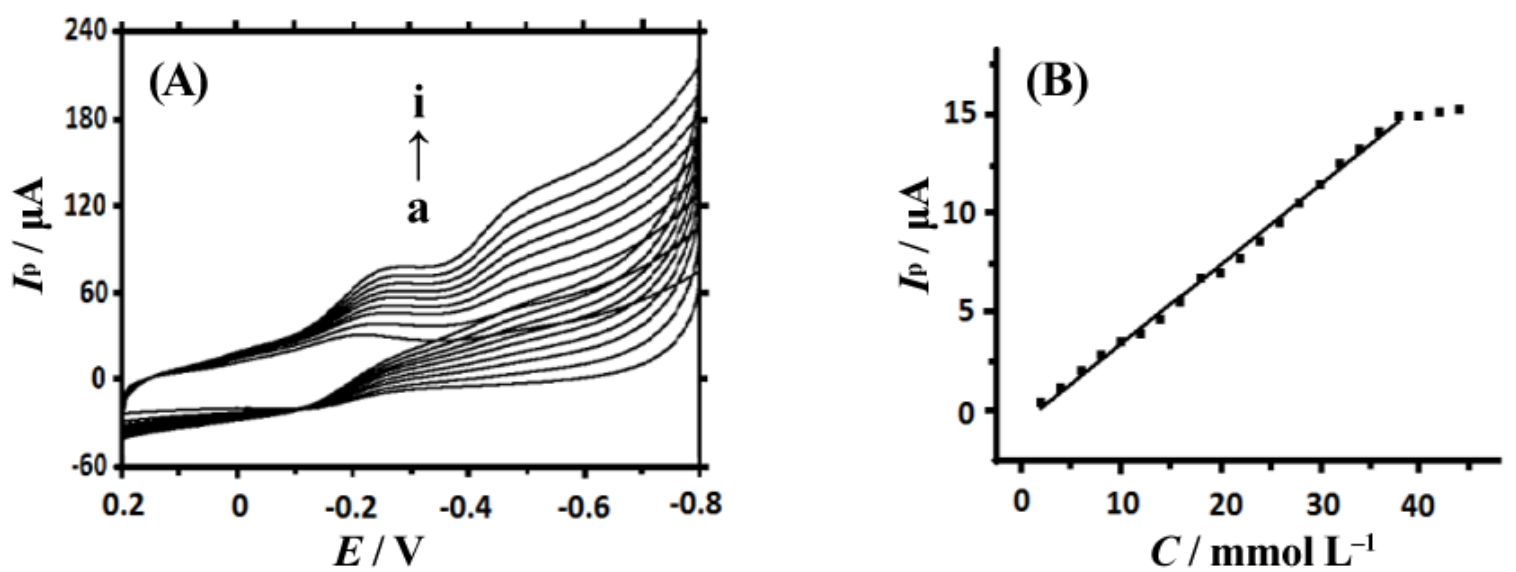

Figure 6. (A) Cyclic voltammograms of CTS/NiO-HRP/CILE in the presence of 2.0, 6.0, 10.0, 14.0, 18.0, 22.0, 26.0, 30.0, 34.0 $\mathrm{mmol} \mathrm{L} \mathrm{L}^{-1} \mathrm{TCA}$ (curves a to i) with the scan rate as $100 \mathrm{mV} \mathrm{s}^{-1}$; (B) the relationship of catalytic reduction peak currents and the TCA concentration.

\section{Electrocatalytic Activity of the HRP Modified Electrode}

TCA is an important target in the fields such as biochemistry and environmental analysis, and the redox proteins modified electrode could be used for the electrocatalysis. [29] Therefore electrocatalytic activity of the HRP modified electrode towards the reduction of TCA was investigated with the typical cyclic voltammograms shown in Figure 6. The addition of different concentrations of TCA resulted in the increase of the reduction peak current at $-0.283 \mathrm{~V}$ with the simultaneously disappearance of the oxidation peak (curves a-i). The further increase of the TCA concentration could result in the appearance of second reduction peak at $-0.512 \mathrm{~V}$, which could be attributed to the formation of a highly reduced form of HRP [HRP Fe(I)] that might dechlorinate di- and mono-chloroacetic acid after the dechlorination of TCA with HRP Fe(II).[30] Therefore the electrocatalytic reaction may be deduced via the following steps, which involved the reduction of HRP Fe(III) to HRP Fe(II), the reduction of TCA with HRP Fe(II) on the electrode, the reduction of HRP Fe(II) to HRP Fe(I) on the electrode, the reduction of di- and mono-chloroacetic acid with HRP Fe(II) and the reoxidation of HRP Fe(I).

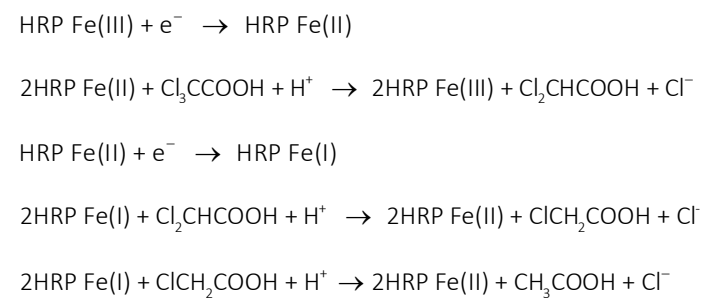

A good linear relationship between the catalytic reduction peak current and TCA concentration was got in the range from $0.8 \mathrm{mmol} \mathrm{L}^{-1}$ to $38.0 \mathrm{mmol} \mathrm{L}^{-1}$ with the linear regression equation as $I_{\mathrm{ss}} / \mu \mathrm{A}=0.40 \mathrm{C} / \mathrm{mmol} \mathrm{L}^{-1}-0.70(\gamma=$ $0.996)$ and the detection limit as $0.26 \mathrm{mmol} \mathrm{L}^{-1}(3 \sigma)$. When the TCA concentration was more than $38.0 \mathrm{mmol} \mathrm{L}^{-1}$, the reduction peak current reached to a plateau, indicating a Michaelis-Menten kinetic for the electrocatalytic reaction. Therefore the apparent Michaelis-Menten constant $\left(K_{\mathrm{M}}^{\mathrm{app}}\right)$ could be calculated from the electrochemical version of the Lineweaver-Burk equation: ${ }^{[31]} 1 / I_{\mathrm{ss}}=\left(1 / I_{\max }\right)\left(1+K_{\mathrm{M}}^{\text {app }} / C\right)$, where $I_{s s}$ is the steady current after the addition of substrate, $C$ is the bulk concentration of the substrate, and $I_{\max }$ is the maximum current measured under saturated substrate condition. The $K_{\mathrm{M}}^{\text {app }}$ value was calculated as 2.062 mmol L-1, which was smaller than that of some previous re-

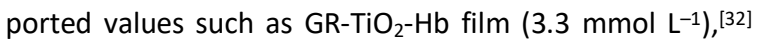
Nafion/ZnWO $4-\mathrm{Hb} / \mathrm{CILE}\left(5.76 \mathrm{mmol} \mathrm{L}^{-1}\right)^{[33]}$ and CTS/GRLDH-Hb/CILE (8.48 mmol L-1). ${ }^{[34]}$ The lower value of $K_{\mathrm{M}}^{\text {app }}$ indicated that HRP immobilized with NiO nanoflower retained its bioactivity and had a high biological affinity to TCA.

\section{Stability and Reproducibility of CTS/NiO-HRP/CILE}

Long-term stability is one of the important parameters of electrochemical biosensor. The HRP modified electrode was stored at $4{ }^{\circ} \mathrm{C}$ refrigerator for a given period to examine the long-term storage stability when not in use. Every 5 days the voltammetric responses of CTS/NiO-HRP/CILE to a $10.0 \mathrm{mmol} \mathrm{L}-1$ TCA solution was examined. Up to 10 days the current response only decreased for about $5.05 \%$ of the initial value. After a 30-day storage period, CTS/NiO$\mathrm{HRP} / \mathrm{ClLE}$ still retained $92.2 \%$ of initial current, which indicated that the modified electrode had a good stability. Therefore $\mathrm{NiO}$ nanoflower was a suitable matrix for the immobilization of HRP and retained its activity due to good biocompatibility. The reproducibility of CTS/NiO-HRP/CILE 
for the determination of $10.0 \mathrm{mmol}^{-1}$ TCA was investigated by fabricating six modified electrodes at the same procedure independently, which showed an acceptable reproducibility with a relative standard deviation (RSD) of $4.3 \%$.

\section{CONCLUSION}

$\mathrm{NiO}$ nanoflower was synthesized and used for the realization of direct electrochemistry of HRP by cyclic voltammetry. A pair of well-defined redox peaks appeared on CTS/NiO-HRP/CILE, which indicated that direct electron transfer of HRP was realized. The result was attributed to large surface area, good biocompatibility and certain conductivity of $\mathrm{NiO}$ nanoflower used. The presence of $\mathrm{NiO}$ nanoflower on the electrode surface resulted in a 3D microstructure with many pores. The immobilized HRP retained its native structure and exhibited excellent electrocatalytic ability to the reduction of TCA with high sensitivity, wide linear range, low detection limit, long-term stability and good reproducibility. Therefore NiO nanoflower has the potential application in constructing third-generation electrochemical biosensor with mediator-free electrochemistry of enzymes.

Acknowledgment. We acknowledge the financial support from the National Natural Science Foundation of China (Nos. 21365010, 21665007), the Science and Research Key Project of Universities of Hainan Province (Hnky2016ZD-10), the International Science and Technology Cooperation Project of Hainan Province (KJHZ2015-13).

\section{REFERENCES}

[1] F. A. Armstrong, H. A. O. Hill, N. J. Walton, Acc. Chem. Res. 1988, 21, 407.

[2] E. Lojou, P. Bianco, Electroanalysis 2004, 16, 1113.

[3] P. A. Prakash, U. Yogeswaran, S. M. Chen, Sensors 2009, 9, 1821.

[4] C. Z. Zhu, G. H. Yang, H. Li, D. Du, Y. H. Lin, Anal. Chem. 2015, 87, 230.

[5] L. Xiang, X. Y. Deng, Y. Jin, Scripta Mater. 2002, 47, 219.

[6] M. S. Niasari, N. Mir, F. Davar, Polyhedron 2009, 28, 1111.

[7] A. Salimi, A. Noorbakhash, E. Sharifi, A. Semnani, Biosens. Bioelectron. 2008, 24, 792.

[8] A. Noorbakhsh, A. Salimi, Biosen. Bioelectron. 2011, 30, 188.
[9] S. Mohan, P. S. N. Maheshwari, S. Sunder, R. Prakach, Analyst 2011, 136, 2845.

[10] A. Salimi, A. Noorbakhsh, H. A. Rafiee-Pour, H. Ghourchian, Electroanal. 2011, 23, 683.

[11] Y. Mu, D. L. Jia, Y. Y. He, Y. Q. Miao, H. L. Wu, Biosen. Bioelectron. 2011, 26, 2948.

[12] M. Opallo, A. Lesniewski, J. Electroanal. Chem. 2011, 656, 2.

[13] N. Maleki, A. Safavi, F. Tajabadi, Anal. Chem. 2006, 78, 3820.

[14] W. Sun, L. F. Li, B. X. Lei, T. T. Li, X. M. Ju, X. Z. Wang, G. J. Li, Z. F. Sun, Mater. Sci. Eng. C. 2013, 33, 1907.

[15] R. F. Gao, J. B. Zheng, Electrochem. Commun. 2009, 11, 1527.

[16] G. N. Li, T. T. Li, Y. Deng, Y. Cheng, F. Shi, W. Sun, Z. F. Sun, J. Solid State Electrochem. 2013, 17, 2333.

[17] S. J. Ding, T. Zhu, J. S. Chen, Z. Y. Wang, C. L. Yuan, X. W. Lou, J. Mater. Chem. 2011, 21, 6602.

[18] W. Sun, M. X. Yang, K. Jiao, Anal. Bioanal. Chem. 2007, 389, 1283.

[19] D. M. Byler, H. Susi, Biopolymers 1986, 25, 469.

[20] J. F. Rusling, T. F. Kumosinski, Intell. Instrum. Comput 1992, 10, 139.

[21] Y. Zhu, G. Cheng, S. Dong, Biophy. Chem. 2002, 97, 129.

[22] W. Sun, D. D. Wang, R. F. Gao, K. Jiao, Electrochem. Commun. 2007, 9, 1159.

[23] A. J. Bard, L. R. Faulkner, Electrochemical methods: fundamentals and applications. Firsted. Wiley, New York, 1980.

[24] S. F. Wang, T. Chen, Z. L. Zhang, X. C. Shen, Z. X. Lu, D. W. Pang, K. Y. Wong, Langmuir 2005, 21, 9260.

[25] E. Laviron, J. Electroanal. Chem. 1979, 101, 19.

[26] W. Sun, L. L. Cao, Y. Deng, S. X. Gong, F. Shi, G. N. Li, Z. F. Sun, Anal. Chim. Acta 2012, 781, 41.

[27] K. J. Huang, Y. X. Miao, L. Wang, T. Gan, M. Yu, L. L. Wang, Process Biochem. 2012, 47, 1171.

[28] J. Yang, Y. Xu, P. G. He, Y. Z. Fang, Electroanalysis 2013, 25, 2345.

[29] N. F. Hu, Pure Appl. Chem. 2001, 72, 1979.

[30] C. H. Fan, Y. Zhuang, G. X. Li, J. Q. Zhu, D. X. Zhu, Electroanalysis 2000, 12, 1156.

[31] R. A. Kamin, G. S. Wilson, Anal. Chem. 1980, 52, 1198.

[32] W. Sun, Y. Q. Guo, X. M. Ju, Y. Y. Zhang, X. Z. Wang, Z. F. Sun, Biosens. Bioelectron. 2013, 42, 207.

[33] C. X. Ruan, Z. L. Sun, J. Liu, J. Lou, W. M. Gao, W. Sun, Y. S. Xiao, Microchim. Acta 2012, 177, 457.

[34] W. Sun, Y. Q. Guo, Y. P. Lu, A. H. Hu, F. Shi, T. T. Li, Z. F. Sun, Electrochim. Acta 2013, 91, 130. 